\title{
Characterizing the Tumor Suppressor Role of CEACAM1 in Multiple Myeloma
}

\author{
Jinge $X u^{a, b, d}$ Bin Liu, Shoubao Ma ${ }^{a, b, c}$ Jubin Zhang ${ }^{a}$ Yuhan Jia Liangjing $X^{a}$ \\ Mingqing Zhu ${ }^{a}$ Suning Chen ${ }^{a}$ Xiaojin Wu ${ }^{a, b, c}$ Depei $W^{a, b, c}$
}

ajiangsu Institute of Hematology, The First Affiliated Hospital of Soochow University, Suzhou, 'Institute of Blood and Marrow Transplantation, Suzhou, 'Collaborative Innovation Center of Hematology, Soochow University, Suzhou, 'The Second Affiliated Hospital of Xuzhou Medical University, Xuzhou, China

\section{Key Words}

CEACAM1 • Multiple $・$ Myeloma $・$ Tumor suppressor

\begin{abstract}
Background/Aims: Carcinoembryonic antigen-related cell adhesion molecule 1 (CEACAM1), also known as CD66a, is a member of the immunoglobulin (Ig) superfamily that belongs to the carcinoembryonic antigen (CEA) family which plays a dual role in cancer. Previous studies showed high expression of CEACAM1 in multiple myeloma (MM). The aim of this study was to investigate the biological consequences of CEACAM1 overexpression in MM. Methods: pEGFPN1-CEACAM1 and pcDNA3.1-CEACAM1 expression plasmids were transfected into U-266 and RPMI8266 cell lines. Effect of CEACAM1 overexpression on the proliferation of two cell lines were tested by the CCK8 assay. Cell cycle and Apoptotic changes after CEACAM1 transfection were examined with AnnexinV-FITC/PI by flow cytometry. Hochest staining assay was used to confirm the apoptotic changes. Caspase-3 activity was examined by Western blotting. The cell invasion and migration activity change after CEACAM1 transfection were performed by well chamber assays and a wound healing, respectively. MMP-2 and MMP-9 proteins expression were detected by Western blotting. Flow cytometry immunophenotyping was be evaluated on myeloma cells from bone marrow taken from 50 patients with symptomatic MM newly diagnosed. The correlations between CEACAM1 expression levels and the clinical features across all groups were investigated. Results: CEACAM1 overexpression significantly suppressed MM cell proliferation, induced cell apoptosis, and inhibited cell invasion and migration possibly through activation of caspase- 3 and downregulation of MMP-2 and MMP9. CEACAM1 expression in patients with DS stage I was more frequent (61.5\%) than those with DS stage II (21.1\%) or III (22.2\%). Furthermore, patients with $\beta 2$-microglobulin levels equal to or less than $3.5 \mathrm{mg} / \mathrm{L}$ had higher CEACAM1 expression than those with $\beta 2$-microglobulin levels greater than $3.5 \mathrm{mg} / \mathrm{L}$. Conclusion: Our findings suggest that CEACAM1 may act as a tumor suppressor in MM.

J. Xu, B. Liu and S. Ma contributed equally to this work.

Xiaojin Wu

and Depei Wu




\section{Introduction}

Carcinoembryonic antigen-related cell adhesion molecule 1 (CEACAM1) is a member of the immunoglobulin (Ig) superfamily that belongs to the carcinoembryonic antigen (CEA) family. It is also known as CD66a and was previously characterized as an adhesion and signaling molecule in epithelial, endothelial, hematopoietic, and tumor cells [1]. CEACAM1 has been implicated in various types of intercellular adhesion and intracellular signaling events important in cell survival, differentiation and growth in both normal and cancer cells $[2,3]$. An interesting feature of CEACAM1 is that it plays a dual role in different cancers. Studies have shown that CEACAM1 expression in colon, hepatoma and prostate cancers can inhibit tumor growth. Loss of gene expression or downregulation has been frequently observed, indicating that it may act as a tumor suppressor [4-8]. CEACAM1 overexpression in thyroid and non-small cell cancer and melanomas are often associated with poor clinical outcome and poor survival [8-11].

In hematologic malignancies, CEACAM1 expression has been reported in childhood acute lymphoblastic leukemia (ALL), chronic myeloid leukemia (CML) and in acute myeloid leukemia (AML) [12]. Ratei et al. reported that CEACAM1 was frequently co-expressed with CD65, CD15, and CD64 in AML [13]. It is also co-expressed, although less frequently, with CD13, CD33, and the two progenitor markers CD34 and CD117. Several studies have shown that CEACAM1 is abnormally expressed on the cell membrane in B-lymphocytes in acute B-lymphoblastic leukemia (B-ALL) [12-14]. Zhao et al. reported that inhibition of CEACAM1 in B-ALL cell lines resulted in reduced cell proliferation and increased percent apoptosis [15]. CEACAM1 expression in multiple myeloma (MM) was first reported by Satoh et al. in 2002 [16]. Lee et al. reported that CEACAM1 expression rate ranged from $69 \%$ to $100 \%$ in plasma cells from five MM patients [17]. However, little is known about the biological consequences of CEACAM1 overexpression in MM.

The objective of the present study was to investigate proliferation, cell cycle progression, apoptosis, migration, and invasion in MM cell lines overexpressing CEACAM1. In addition, CEACAM1 expression was evaluated by flow cytometry analysis on myeloma cells from bone marrow from 50 patients with MM. Possible relationships between CEACAM1 expression and patient characteristics were investigated.

\section{Materials and Methods}

Cell lines and culture

Two human myeloma cell lines (U-266 and RPMI8266) were obtained from Jiangsu Institute of Hematology (Jiangsu, China). The cells were cultured in RPMI 1640 (Hyclone, USA) with 10\% fetal bovine serum (Gibco, USA) and maintained at $37^{\circ} \mathrm{C}$ with $5 \% \mathrm{CO}_{2}$.

\section{Plasmid construction and transfection}

Full-length CEACAM1 cDNA was amplified by PCR, and was subcloned into pEGFP-N1 or pcDNA3.1 vectors. The primers used for amplifying full length CEACAM1 ORF were as follows: forward: 5'-GCCAGCAGGAGACACCAT-3'; and reverse: 5'-TCATCTTGTTAGGTGGGTCATTG-3'. An empty expression plasmid of the same type was used as a control. All constructed plasmids were confirmed by DNA sequencing. Lipofectamine 2000 (Invitrogen, USA) was used for plasmid transfection according to the manufacturers' instructions. Cells were selected using selection media G418 (Invitrogen, USA). The transfected cells were maintained in RPMI 1640 medium with $10 \%$ fetal bovine serum, penicillin $(10 \mathrm{U} / \mathrm{ml})$, and streptomycin (10 $\mathrm{mg} / \mathrm{ml})$.

\section{In vitro Cell Viability Assay}

The stably transfected cells were seeded into 96-well plates (10, 000 cells / well), and maintained in RPMI 1640 as described above. Ten microliters of CCK-8 solution was added in each well. The plates were incubated for 3 hours and sampled at $0,24,48$, or $72 \mathrm{hr}$ time points afterwards. The absorbance was measured at $450 \mathrm{~nm}$ and normalized to the cell counts. 


\section{Cellular Physiology Cell Physiol Biochem 2018;45:1631-1640 \begin{tabular}{l|l|l|l} 
DOI: 10.1159/000487730 & O 2018 The Author(s). Published by S. Karger AG, Basel \\
www.karger.com/cpb
\end{tabular}

RNA extraction and RT-PCR.

Total RNA was extracted using Nucleic acid PrepStation from Tiangen Biotech (Beijing, China) according to the manufacturer's instructions. The concentration, quality, and purity of RNA were measured by a UV spectrophotometer at 260 / $280 \mathrm{~nm}$. cDNA synthesis was performed using High Capacity cDNA Reverse Transcription Kit (Life Technologies, USA) following manufacturer's instructions. The mixture was then incubated at $42^{\circ} \mathrm{C}$ for $1 \mathrm{~h}$. The enzyme was inactivated by heating at $95^{\circ} \mathrm{C}$ for $5 \mathrm{~min}$. The primer sequences used for PCR amplification were as follows: 5'- CTCTCCTGCTATGCAGCCTC-3' (reverse primer) and 5' - ACTGAGTTATTGGCGTGGCA-3' (forward primer) for CEACAM1; 5'- CTGTCACCTTCACCGTTCCAGTTT -3' (reverse primer) and 5'- CTTAGTTGCGTTACACCCTTTCTTG -3' (forward primer) for ß-actin. Quantitative reverse transcription-polymerase chain reaction (qRT-PCR) was performed in a total volume of $20 \mu \mathrm{L}$, containing 50 ng cDNA, $1 \mu \mathrm{M}$ of each primer, $1 \mu \mathrm{M}$ SYBR Green, and $10 \mu \mathrm{L}$

$2 \times$ Taq PCR Master-mix. Amplification was performed in an Exicycler TM 96 qPCR system. The amplification conditions included a denaturation step at $95^{\circ} \mathrm{C}$ for 5 minutes followed by 40 cycles of 15 seconds at $95^{\circ} \mathrm{C}, 45$ seconds at $58^{\circ} \mathrm{C}$ to $60^{\circ} \mathrm{C}$, and 45 seconds at $72^{\circ} \mathrm{C}$. A melting curve cycle was carried out as the last step. The data was analyzed using ExicyclerTM 96 software. Samples were tested in triplicates and the mean cycle numbers were included in the analysis. The relative fold change was normalized against $\beta$-actin. The CEACAM1 mRNA expression relative to $\beta$-actin was calculated using the $2^{-\Delta \Delta C T}$ method.

\section{Assay for CEACAM1-induced apoptosis}

Apoptotic cell death was evaluated using a FITC-annexin V/propidium iodide (PI) double-staining kit (KeyGEN Biotech, Nanjing, China) following manufacturer's instructions. Briefly, the cells were washed with PBS and resuspended in $500 \mu \mathrm{L}$ binding buffer with $5 \mu \mathrm{L}$ annexin $\mathrm{V}$ and $10 \mu \mathrm{L}$ propidium iodide. The apoptosis assay was performed $48 \mathrm{hr}$ after transfection. Stained cells were analyzed by flow cytometry. Hoechst Staining Kit (Beyotime, Shanghai, China) was also used to confirm apoptotic changes in the cell lines after transfection. The fluorescence microscopy showed the presence of typical apoptotic bodies as dense particles emitting bright blue fluorescence signals indicative of pyknosis in both cell lines. Experiments were repeated independently at least three times using both methods and similar results were obtained.

\section{Cell cycle analysis by flow cytometry}

After a $48 \mathrm{hr}$ transfection period, cells were washed with PBS and fixed in $75 \%$ ethanol at $4{ }^{\circ} \mathrm{C}$ overnight. After a $24 \mathrm{hr}$ fixation period, the cells were washed with PBS and treated with propidium iodide (PI) stain (KeyGEN Biotech, Nanjing, China) for $30 \mathrm{~min}$. Then stained cells were analyzed by flow cytometry.

\section{Cell migration and invasion assays}

To measure the cell invasion activity, trans-well chamber assays were performed using a BD BioCoat Matrigel Invasion Chamber (BectonDickinson, MA). Cells were resuspended in serum-free RPMI-1640 and then added to the upper chamber at a density of $5 \times 10^{4}$ cells/insert. RPMI-1640 containing 10\% FBS was added to the lower chamber. After incubation at $37^{\circ} \mathrm{C}$ for $24 \mathrm{hr}$, the cells that invaded into the lower chamber were counted. To measure the cell migration activity, trans-well chamber assays were performed using BD BioCoat Cell Culture Inserts (Becton-Dickinson) following a protocol similar to that described above for the cell invasion assay. A wound healing assay was also performed to determine the migration activity. The monolayer was scratched with a pipette tip. The cultures were then incubated for $24 \mathrm{hr}$ and the number of cells migrating over the exposed dish surface were recorded.

\section{Western blotting analysis}

Whole cell extracts were prepared using the M-PER reagent (Life Technologies, Rockford, USA). Proteins were separated by SDS-PAGE (Bio-Rad Laboratories), transferred to polyvinylidene fluoride membranes (Millipore, USA), blocked for 1 hour in buffer containing 5\% nonfat dry milk (Lab Scientific), and incubated with the primary antibody overnight at 1/100 dilution. Rabbit anti Caspase-3 polyclonal antibody (Catalog number: AC033), rabbit anti MMP-2 polyclonal antibody (Catalog number: AF0234), and rabbit anti Cyclin D1 polyclonal antibody (Catalog number: AF0126) were purchased from Beyotime (Shanghai, China). Anti-CEACAM1 rabbit polyclonal antibody (Catalog number: D120408) was purchased from Sangon biotech (Shanghai, China). Rabbit anti MMP-9 polyclonal antibody (Catalog number: 3582) was purchased from Cell Signaling Technology (Boston, MA, USA). Mouse anti $\beta$-actin (Catalog number: AF0003) was purchased from Beyotime (Shanghai, China), and used at a 1/1, 000 dilution. Secondary antibodies (IRDye 800CW and IRDye 680LT) purchased from LI-COR(Lincoln,USA ) were used at 1/5, 000 dilution. Secondary an- 
tibody was applied the next day and the signal was visualized on a Molecular Imager ChemidocXRS (Bio-Rad Laboratories, California, USA) using the Pierce Supersignal West Pico chemiluminescent substrate (Life Technologies, Rockford, USA). Images were obtained using Quantity One software (Bio-Rad Laboratories).

\section{Patient samples}

Bone mamples used in this study were taken from 50 patients with symptomatic MM newly diagnosed following diagnostic criteria published by the World Health Organization in 2008. The demographic and clinical data from the patients are summarized in Table 1. The samples were collected between June and October 2015. Median and mean age of the 29 male and 21 female patients was 64 and 63.5 years, respectively. javascript:void(0);This investigation was approved by the Ethics Committee of the Institutional Review Board of The First Affiliated Hospital of Soochow University (Jiangsu, Public Republic of China).

\section{CEACAM1 expression by flow cytometry analysis}

Flow cytometry immunophenotyping was performed on myeloma cell populations using a Navios flow cytometer (Beckman Coulter, Inc, USA). All cases were tested using a panel of antibodies raised against leukocyte and plasmacyte-associated markers including surface fluorochrome-conjugated antibodies against CEACAM1, CD45, CD19, CD38, CD56, CD138, CD7, CD34, CD10, CD20, CD13, CD14, CD33, CD15, CD117 and HLA-DR (Beckman Coulter, Inc, Indianapolis, USA). Surface antigen expression was considered positive when over $20 \%$ of the plasmacytes were positive for the signal. The cells with the $\mathrm{CD} 38^{++} / \mathrm{CD} 138^{+} /$ CD45 /CD19- combination were categorized as myeloma cells and those with the CD38 ${ }^{++} / \mathrm{CD}^{-} 6^{-} / \mathrm{CD} 45^{-/+} /$ $\mathrm{CD} 19^{+}$combination were categorized as normal plasma cells. Isotype-matched negative controls were used in all assays to distinguish positive from negative cells. Flow cytometry results were processed using Kaluza software, version 1.3 (Beckman Coulter, Inc.).

\section{Statistical Analysis}

SPSS software version 16.0 for Windows (SPSS Inc., Chicago, IL, USA) was used for all analyses. The correlations between CEACAM1 expression levels and the clinical features across all groups were investigated using Fisher's exact test for categorical data and the nonparametric Mann-Whitney U test for continuous variables. For the quantitative data javascript:void(0); comparisons between treated and control groups, a paired t-test or two-way ANOVA analysis was performed. A $P$ value less than 0.05 was considered statistically significant.

\section{Results}

Effect of CEACAM1 overexpression on the proliferation of U266 and RPMI 8266 cell lines peGFP-N1-CEACAM1 and pcDNA3.1-CEACAM1 expression plasmids were constructed and successfully transfected into the U266 and RPMI8266 cell lines, respectively (Fig. 1a 
,1b and 1c). Transfection efficiency was approximately $70 \%$. The CCK8 assay results showed that the cell count in U-266 and RPMI8266 cell lines were both reduced $48 \mathrm{~h}$ after CEACAM1 transfection. (Fig. 1d).

\section{CEACAM1 overexpression in MM cells induced apoptosis possibly via increasing caspase-3 activity and affecting cell cycle progression by down-regulating cyclinD1 Both pcDNA3.1-} CEACAM1 stably transfected and non-transfected U-266 and RPMI8266 cell lines were incubated for $48 \mathrm{hr}$ and then examined for the cells positive for AnnexinV-FITC/PI to determine apoptosis by flow cytometry. Apoptosis rates after CEACAM1 transfection were increased in both U-266 and RPMI8266 compared to the non-transfected cells (Fig. 2a). We also used Hochest staining assay to confirm the apoptotic changes in the two cell lines after transfection. The fluorescence microscopy showed the presence of typical apoptotic bodies as dense particles emitting bright blue fluorescence due to pyknosis in both U-266 and RPMI8266 cells (Fig. 2b). Western blotting analysis showed that caspase-3, the main terminal enzyme in the apoptotic process, was activated after CEACAM1 transfection in both U-266 and RPMI8266 cell lines (Fig. 2d).

Cell cycle analysis after transfection showed a marked growth arrest in the G1 phase in both cell lines. Compared to the control group, CEACAM1 transfection significantly reduced the number of cells in the S phase, with no significant impact on the number of cells in the G2 cycle (Fig. 2c). Western blotting analysis showed that cyclinD1 was inhibited after CEACAM1 transfection in both cell lines (Fig. 2d).

\section{CEACAM1 expression inhibits cell invasion and migration}

In the wound healing assay, the wounded area in the U-266 and RPMI8266 cell lines began to close at $12 \mathrm{hr}$ and was covered with migrating cells within $24 \mathrm{hr}$, whereas the cells transfected with pcDNA3.1-CEACAM1/U266 and pcDNA3.1-CEACAM1/RPMI8266 had more uncovered areas at $24 \mathrm{hr}$ (Fig. 3a). The number of invading cells in cells transfected with pcDNA3.1-CEACAM1/U266 and pcDNA3, 1-CEACAM1/RPMI8266 were lower than those 
in the control cell lines (Fig. 3b). Transfection decreased the expression of matrix metalloproteinase-2 (MMP-2) and matrix metalloproteinase-9 (MMP-9) proteins, the two very important mediators of tumor migration (Fig. $3 \mathrm{c}$ and $3 \mathrm{~d}$ ).

\section{CEACAM1 expres- sion in $M M \quad p a-$ tients is associ- ated with disease stage (DS) and $\beta 2$ - microglobulin ex- pression}

We also compared CEACAM1 expression in the healthy subjects and MM patients with different $\beta 2$ microglobulin levels. CEACAM1 expression was $3 \%$ in the healthy subjects, $\quad 31.45 \%$ in the patients with $\beta 2$ microglobulin greater than $3.5 \mathrm{mg} / \mathrm{L}$ and $96.89 \%$ in the patients with $\beta 2$-microglobulin levels less or equal to $3.5 \mathrm{mg} / \mathrm{L}$ (Fig. 4).

Positive CEACAM1 expression was more frequent in DS stage I than the DS stage II or III groups of patients $(P$ $<0.05$ ). However, there were no significant correlations between CEACAM1 expression levels and the age, gender, percentage of bone marrow myeloma cells, chromosome karyotype, type of light chain, M-protein, IgH rearrangement, or renal function (Table 1).

\section{Discussion}

CEACAM1 was originally discovered in the bile, but a subsequent immunohistochemical study disclosed a wide tissue distribution [11]. With regard to hematopoietic tissues, its expression has been reported in granulocytes, lymphocytic tumor cells, activated T lymphocytes, natural killer (NK) cells, and intestinal intraepithelial lymphocytes, suggesting that

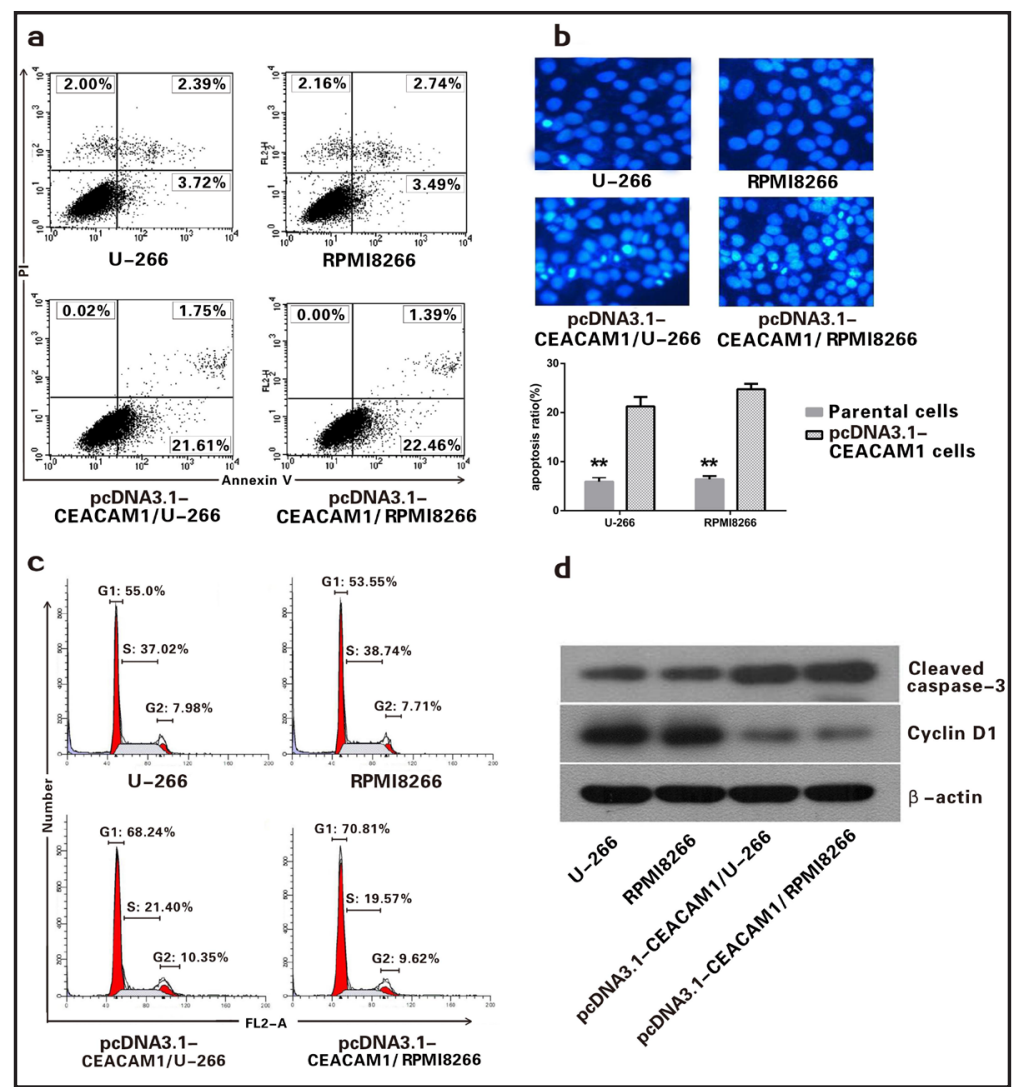

Fig. 2. Effect of CEACAM1 overexpression on the apoptosis and cell cycle in U-266 and RPMI8266 cell lines. a, Cells were incubated for $48 \mathrm{hr}$, then examined by flow cytometry for apoptosis by determining the number of cells positive for AnnexinV-FITC/PI. The apoptosis rate in the U-266 and RPMI8266 after CEACAM1 transfection were both increased compared to the control. b, Hochest staining assay was used to confirm the apoptotic changes in the U-266 and RPMI8266 cell lines after transfection with CEACAM1 pcDNA3.1 vectors. Compared to the parental cells, the apoptosis ratio were elevated in both cell lines after transfection. The $\left.{ }^{* *}\right)$ denotes $\mathrm{P}<0.01 \mathrm{c}$, Cell cycle analysis after CEACAM1 transfection showed a marked growth arrest in the G1 phase in both cell lines. Compared to the control group, CEACAM1 transfection significantly reduced the cell number in $\mathrm{S}$ phase, with no significant impact on the number of cells in the G2 cycle. d, western blotting analysis showed that caspase- 3 was activated but cyclinD1 was inhibited after CEACAM1 transfection in both U-266 and RPMI8266 cell lines. 
Fig. 3. CEACAM1 inhibits cell invasion and migration. a, In the wound healing assay, the wounded area in the U-266 and RPMI8266 cell lines began to close at $12 \mathrm{hr}$ and was covered with migrating cells within 24 hr, while the pcDNA3.1CEACAM1/U-266 and p c D NA3. 1 - CEAC AM 1/ RPMI8266 transfected cells left more uncovered area at $24 \mathrm{hr}$. b, The number of invading cells were lower in the transfected pcDNA3.1CEACAM1/U-266 and pcD NA3. 1 - CEACAM 1 / RPMI8266 than in the control cell lines. The $\left(^{* *}\right)$ denotes $\mathrm{P}<0.01$ vs. control. c, MMP-2 and MMP-9 protein levels in cells transfected with pcDNA3.1CEACAM1/U-266 and pcDNA3.1 - CEACAM 1 /

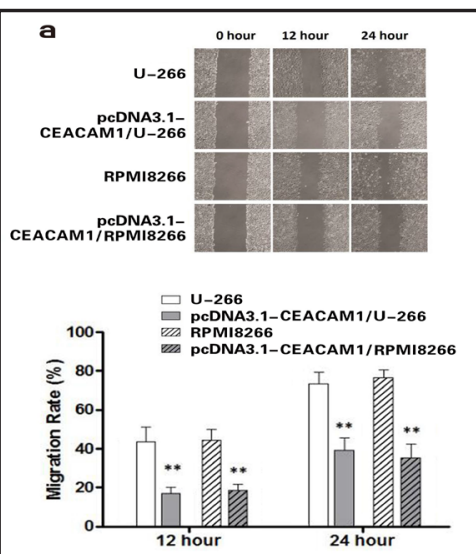

c
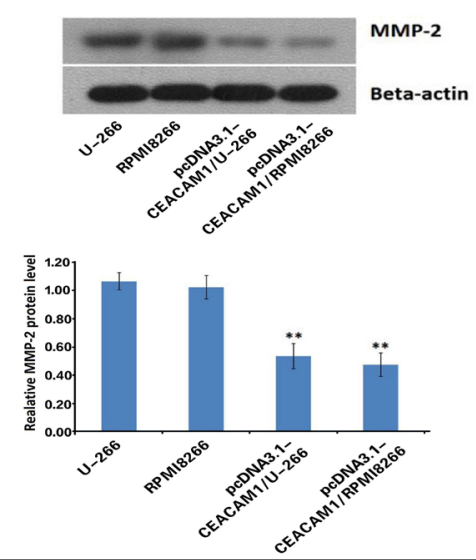

b
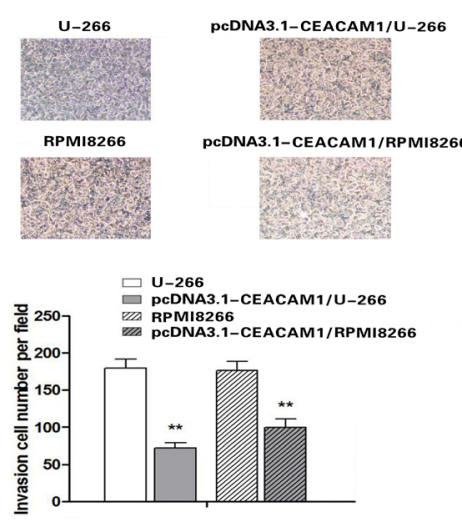

d

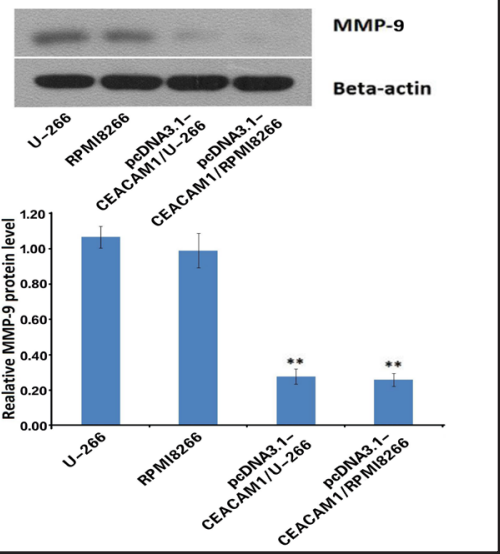

RPMI8266 were lower

compared to those in the control cell lines $(\mathrm{P}<0.001)$. $d$, MMP-9 protein levels in the cells transfected with pcDNA3.1-CEACAM1/U-266 and pcDNA3.1-CEACAM1/RPMI8266 were lower compared to the control cell lines $(\mathrm{P}<0.001)$.

Fig. 4. Flow cytometric analysis of CEACAM1 expression on the surface of plasma cells from a healthy individual and myeloma cells from two multiple myeloma patients. All cells were aspirated from the bone marrow. The combination CD38++/CD138+/CD45-/ CD19- was used for defining myeloma cells and the combination CD38++/CD56-/CD45

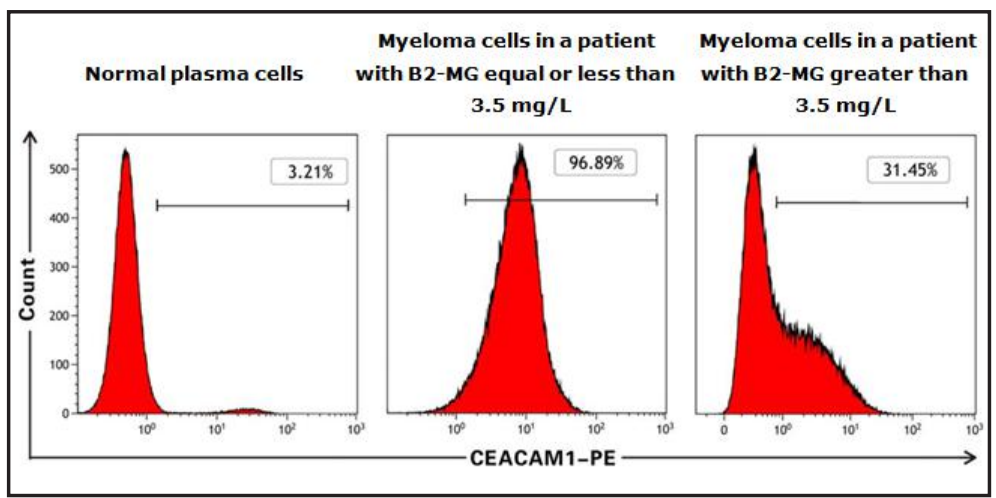

${ }^{+} / \mathrm{CD} 19+$ was used for defining

normal plasma cells. Lower $\beta 2$-MG levels were associated with higher CEACAM1 expression in myeloma cells $(\mathrm{P}<0.01)$. Abbreviation used: $\beta 2-\mathrm{MG}=\beta 2$-microglobulin.

CEACAM1 may play an important role in normal and transformed lymphocyte functions [3]. Expression of CEACAM1 in multiple myeloma was first discovered by Satoh et al. in 2002 [16]. Previously, others showed that CEACAM1 is down-regulated or dysregulated in carcinomas [18]. For example, in prostate cancer, CEACAM1 is lost during the progression from Gleason stage 3 to $4[19,20]$. It is also down-regulated in colon carcinomas, hepatocellular KARGER 
carcinomas [21], and in a proportion of breast cancers. However, it is known for some time that the down-regulation of CEACAM1 is not a general phenomenon in epithelial malignancies [19]. For example, it is up-regulated in gastric and thyroid cancers and metastasing malignant melanomas [22]. Similarly, in non-small cell lung cancer, CEACAM1 is up-regulated and it is an indicator of a poor prognosis $[23,24]$. Therefore, CEACAM1 may have different roles in different types of cancers [6]. The role of CEACAM1 in MM has not been well investigated in the literature.

Our investigation found that CEACAM1 expression was more frequent in DS stage I than DS stage II or III groups of patients. In addition, CEACAM1 expression was more frequent in patients with $\beta 2$-microglobulin levels less than or equal to $3.5 \mathrm{mg} / \mathrm{L}$ than those with levels greater than $3.5 \mathrm{mg} / \mathrm{L}$. In 2002, Satoh et al., reported that 18 of $21 \mathrm{MM}$ cases (86\%) were CEACAM1 positive whereas our results show that only 16 of $50 \mathrm{MM}$ cases (32\%) were positive. The most likely reason for this discrepancy could be that the patients analyzed by Satoh et al.and in our study were in different disease stages.

Our observation that the patients with DS I had more frequent CEACAM1 expression than those in DS II or III suggest that CEACAM1 is probably a marker for better prognosis in MM. To further investigate this phenomenon, we transfected full-length CEACAM1 expression plasmids into the U-266 and RPMI8266 cell lines and investigated the effects of CEACAM1 overexpression on cell proliferation, cell cycle, apoptosis, and cell migration and invasion in vitro.

After transfection, the cell count decreased and apoptosis increased in both cell lines compared to the non-transfected controls. These findings suggest that CEACAM1 overexpression could suppress MM cell proliferation and induce apoptosis. Cell cycle analysis after transfection showed a marked growth arrest in the G1 phase in both cell lines. Compared to the control group, CEACAM1 transfection significantly reduced the cell number in S phase, with no significant impact on the number of cells in G2 phase. The fluorescence microscopy showed that the typical apoptotic bodies were more obvious in the U-266 and RPMI8266 cell lines after CEACAM1 transfection, and the western blotting analysis showed that caspase- 3 expression increased after the transfection in both cell lines. Therefore, the decrease in cell proliferation and increase in apoptosis seen in MM cells overexpressing CEACAM1 in our study maybe due to the caspase-3 overexpression.

In our study, CEACAM1 overexpression resulted in less MMP-2 and MMP-9 protein expression and lower invasion and migration compared to the control cell lines. These results suggest that decreased invasion and migration in MM cells could be due to downregulation of MP-2 and MP-9 protein expression. Ebrahimnejad et al., on the other hand, reported that CEACAM1 enhanced invasion and migration in melanocytic and melanoma cells. The authors concluded that CEACAM1 actively contributed to tumor progression [25]. The reason for this discrepancy warrants further investigation but could be that CEACAM1 may have different roles in different cancers as discussed above [26, 27].

\section{Conclusion}

Our data show that CEACAM1 overexpression in MM cell lines exerts an anti-tumor effect, suggesting that CEACAM1 may be acting as a tumor-suppressor in MM. To our knowledge, this is the first study clarifying the functional role of CEACAM1 in MM in vitro.

\section{Acknowledgements}

This work was supported by the National Natural Science Foundation of China (No.81470346, 81773361), the Priority Academic Program Development of Jiangsu Higher Education Institutions (PAPD), National Clinical Key Specialty Development Project and National Public Health Grand Research Foundation (201202017), the Network Hospital 


\section{Cellular Physiology Cell Physiol Biochem 2018;45:1631-1640

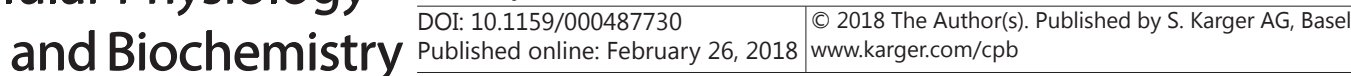 \\ Xu et al.: CEACAM1 in Multiple Myeloma}

of Clinical Medicine Science and Technology Project, Jiangsu Science and Technology Department (BL2012005), Jiangsu Natural Science Foundation (BK20161204), Jiangsu "333" talent project (BRA2015497) , the national key research and development program (2016YFC0902800), Innovation Capability Development Project of Jiangsu Province (BM2015004), Six Talent Peaks Project in Jiangsu Province and Jiangsu Provincial Medical Youth Talent (QNRC2016707).

\section{Disclosure Statement}

Authors report no conflicts of interest.

\section{References}

1 Prall F, Nollau P, Neumaier M, Haubeck HD, Drzeniek Z, Helmchen U, Loning T, Wagener C: CD66a (BGP), an adhesion molecule of the carcinoembryonic antigen family, is expressed in epithelium, endothelium, and myeloid cells in a wide range of normal human tissues. J Histochem Cytochem 1996;44:35-41.

-2 Abou-Rjaily GA, Lee SJ, May D, Al-Share QY, Deangelis AM, Ruch RJ, Neumaier M, Kalthoff H, Lin SH, Najjar SM: CEACAM1 modulates epidermal growth factor receptor--mediated cell proliferation. J Clin Invest 2004;114:944-952.

3 Skubitz KM, Skubitz AP: Interdependency of CEACAM-1, -3, -6, and -8 induced human neutrophil adhesion to endothelial cells. J Transl Med 2008;6:78.

4 Cruz PV, Wakai T, Shirai Y, Yokoyama N, Hatakeyama K: Loss of carcinoembryonic antigen-related cell adhesion molecule 1 expression is an adverse prognostic factor in hepatocellular carcinoma. Cancer 2005;104:354-360.

-5 Brummer J, Neumaier M, Gopfert C, Wagener C: Association of pp60c-src with biliary glycoprotein (CD66a), an adhesion molecule of the carcinoembryonic antigen family downregulated in colorectal carcinomas. Oncogene 1995;11:1649-1655.

-6 Hokari M, Matsuda Y, Wakai T, Shirai Y, Sato M, Tsuchiya A, Takamura M, Yamagiwa S, Suzuki K, Ohkoshi S, Ichida T, Kawachi H, Aoyagi Y: Tumor suppressor carcinoembryonic antigen-related cell adhesion molecule 1 potentates the anchorage-independent growth of human hepatoma HepG2 cells. Life Sci 2007;81:336345.

7 Kammerer R, Riesenberg R, Weiler C, Lohrmann J, Schleypen J, Zimmermann W: The tumour suppressor gene CEACAM1 is completely but reversibly downregulated in renal cell carcinoma. J Pathol 2004;204:258267.

8 Kuespert K, Pils S, Hauck CR: CEACAMs: their role in physiology and pathophysiology. Curr Opin Cell Biol 2006;18:565-571.

-9 Oliveira-Ferrer L, Tilki D, Ziegeler G, Hauschild J, Loges S, Irmak S, Kilic E, Huland H, Friedrich M, Ergun S: Dual role of carcinoembryonic antigen-related cell adhesion molecule 1 in angiogenesis and invasion of human urinary bladder cancer. Cancer Res 2004;64:8932-8938.

10 Riethdorf L, Lisboa BW, Henkel U, Naumann M, Wagener C, Loning T: Differential expression of CD66a (BGP), a cell adhesion molecule of the carcinoembryonic antigen family, in benign, premalignant, and malignant lesions of the human mammary gland. J Histochem Cytochem 1997;45:957-963.

11 Obrink B: On the role of CEACAM1 in cancer. Lung Cancer 2008;60:309-312.

-12 Carrasco M, Muñoz L, Bellido M, Bernat S, Rubiol E, Ubeda J, Sierra J, Nomdedéu JF:CD66 expression in acute leukaemia. Ann Hematol 2000;79:299-303.

13 Ratei R, Karawajew L, Schabath R, Ehrfeldt A, Grunert F, Ludwig WD: Differential expression of the carcinoembryonic antigen-related cell adhesion molecules panCD66, CD66a, CD66c and of sialyl-Lewis $\mathrm{x}$ (CD15s) on blast cells of acute leukemias. Int J Hematol 2008;87:137-143.

14 Hanenberg H, Baumann M, Quentin I, Nagel G, Grosse-Wilde H, von Kleist S, Gobel U, Burdach S, Grunert F: Expression of the CEA gene family members NCA-50/90 and NCA-160 (CD66) in childhood acute lymphoblastic leukemias (ALLs) and in cell lines of B-cell origin. Leukemia 1994;8:2127-2133. 


\section{Cellular Physiology Cell Physiol Biochem 2018;45:1631-1640

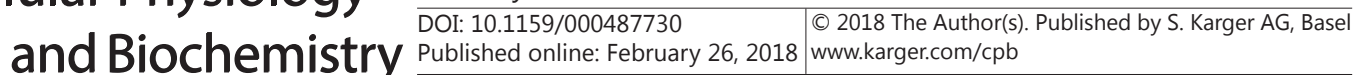 \\ Xu et al.: CEACAM1 in Multiple Myeloma}

15 Zhao W, Zhang Y, Liu D, Zhong L, He Q, Zhao Y: Abnormal expression of CD66a promotes proliferation and inhibits apoptosis of human leukemic B cells in vitro. Leuk Lymphoma 2015;56:202-210.

16 Satoh Y, Hayashi T, Takahashi T, Itoh F, Adachi M, Fukui M, Kuroki M, Kuroki M, Imai K, Hinoda Y: Expression of CD66a in multiple myeloma. J Clin Lab Anal 2002;16:79-85.

17 Lee C, Guinn BA, Brooks SE, Richardson D, Orchard K: CD66a (CEACAM1) is the only CD66 variant expressed on the surface of plasma cells in multiple myeloma: a refined target for radiotherapy trials? $\mathrm{Br} \mathrm{J}$ Haematol 2010;149:795-796.

18 Ling Y, Wang J, Wang L, Hou J, Qian P, Xiang-dong W: Roles of CEACAM1 in cell communication and signaling of lung cancer and other diseases. Cancer Metastasis Rev 2015;34:347-357.

19 Beauchemin N, Arabzadeh A: Carcinoembryonic antigen-related cell adhesion molecules (CEACAMs) in cancer progression and metastasis. Cancer Metastasis Rev 2013;32:643-671.

20 Tilki D, Irmak S, Oliveira-Ferrer L, Hauschild J, Miethe K, Atakaya H, Hammerer P, Friedrich MG, Schuch G, Galalae R, Stief CG, Kilic E, Huland H, Ergun S: CEA-related cell adhesion molecule-1 is involved in angiogenic switch in prostate cancer. Oncogene 2006;25:4965-4974.

21 Wu CC, Yang SF, Chen WT, Tsai HP, Luo CW, Chai CY, Yin HL: Absence of CD66a expression is associated with high microvessel density and high histologic grade in hepatocellular carcinoma. Kaohsiung J Med Sci 2016;32:306-312.

22 Nittka S, Gunther J, Ebisch C, Erbersdobler A, Neumaier M: The human tumor suppressor CEACAM1 modulates apoptosis and is implicated in early colorectal tumorigenesis. Oncogene 2004;23:9306-9313.

-23 Laack E, Nikbakht H, Peters A, Kugler C, Jasiewicz Y, Edler L, Brummer J, Schumacher U, Hossfeld DK: Expression of CEACAM1 in adenocarcinoma of the lung: a factor of independent prognostic significance. J Clin Oncol 2002;20:4279-4284.

-24 Klaile E, Klassert TE, Scheffrahn I, Muller MM, Heinrich A, Heyl KA, Dienemann H, Grunewald C, Bals R, Singer BB, Slevogt H: Carcinoembryonic antigen (CEA)-related cell adhesion molecules are co-expressed in the human lung and their expression can be modulated in bronchial epithelial cells by non-typable Haemophilus influenzae, Moraxella catarrhalis, TLR3, and type I and II interferons. Respir Res 2013;14:85.

25 Ebrahimnejad A, Streichert T, Nollau P, Horst AK, Wagener C, Bamberger AM, Brummer J: CEACAM1 enhances invasion and migration of melanocytic and melanoma cells. Am J Pathol 2004;165:1781-1787.

-26 Arabzadeh A, Dupaul-Chicoine J, Breton V, Haftchenary S, Yumeen S, Turbide C, Saleh M, McGregor K, Greenwood CM, Akavia UD, Blumberg RS, Gunning PT, Beauchemin N: Carcinoembryonic Antigen Cell Adhesion Molecule 1 long isoform modulates malignancy of poorly differentiated colon cancer cells. Gut 2016;65:821-829.

27 Ieda J, Yokoyama S, Tamura K, Takifuji K, Hotta T, Matsuda K, Oku Y, Nasu T, Kiriyama S, Yamamoto N, Nakamura Y, Shively JE, Yamaue H: Re-expression of CEACAM1 long cytoplasmic domain isoform is associated with invasion and migration of colorectal cancer. Int J Cancer 2011;129:1351-1361. 